J. Lake Sci. (湖泊科学), 2019, 31(3): 814-824

DOI 10. 18307/2019. 0319

(C) 2019 by Journal of Lake Sciences

\title{
鄱阳湖植食越冬候鸟粪便对洲滩湿地薹草枯落物分解过程及碳、氮、 磷释放的影响
}

张全军 ${ }^{1,5}$, 张广帅 ${ }^{2,3}$, 万松贤 ${ }^{4}$, 刘 宇 $^{1}$, 夏少霞 ${ }^{1}$,李 雅 $^{1,5}$, 许 策 $^{1,5}$, 于秀波 ${ }^{1,5 * *}$

(1: 中国科学院地理科学与资源研究所,中国科学院生态系统网络观测与模拟重点实验室, 北京 100101)

(2: 国家海洋环境监测中心, 大连 116023)

(3: 国家海洋局海域管理技术重点实验室,大连 116023)

(4:江西鄱阳湖南矶湿地国家级自然保护区管理局,南昌 330038)

(5: 中国科学院大学, 北京 100049)

摘 要: 于 2017 年 1 月- 2017 年 6 月采用分解袋技术和鸟粪添加试验研究了鄱阳湖湿地植食性越冬水鸟粪便和典型洲 滩湿地植物菫草枯落物分解过程中碳、氮、磷释放动态的差异性以及植食性越冬水鸟粪便的加人对翻阳湖湿地莒草分解 过程的影响. 结果表明, 不同分解时间内荎草枯落物、䔔草+鸟粪中菫草枯落物和鸟粪 3 种样品的干物质损失率和分解速

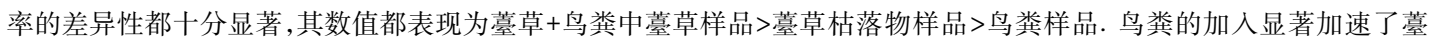
草分解进程,使其平均分解速率提升了约 $21.23 \%$. Olson 负指数模型模拟预测显示, 菫草+鸟粪中荎草、荎草枯落物和鸟粪 样品分解完成 $50 \%$ 所需的时间依次为 $259 、 314$ 和 355 天,分解完成 $95 \%$ 所需的时间大约依次约为 $3.1 、 3.7$ 和 4.2 年. 3 种 样品碳、氮、磷都表现为净释放模式, 其相对回归指数之间差异性也显著. 碳相对归还指数都表现为荎草 + 鸟粪中薹草 $>$ 荎 草枯落物 $>$ 鸟粪, 氮相对归还指数和磷相对归还指数值都表现为鸟粪 $>$ 荎草 + 鸟粪中茥草 $>$ 䔔草枯落物. 本研究推测鸟粪添 加给分解环境带来额外的营养物质, 使分解者微生物的数量、种类、活性增加以及鸟粪刺激产生了一系列降解酶, 如木质 素酶、纤维素酶、蔗糖酶等,胞外酶数量和活性增加, 是鸟粪促进莒草分解的关键因素, 而样品中初始养分含量以及分解 过程碳、氮、磷化学计量比的变化是造成其分解速率和养分释放差异显著的主要原因.

关键词: 鄱阳湖; 越冬水鸟; 湿地植物; 分解过程; 湿地碳、氮、磷生物地球化学循环

\section{Effects of herbivorous overwintering migratory birds' droppings on the decomposition of Carex cinerascens Kükenth and C, N, P release in Lake Poyang wetland}

\author{
ZHANG Quanjun $^{1,5}$, ZHANG Guangshuai ${ }^{2,3}$, WAN Songxian ${ }^{4}$, LIU Yu ${ }^{1}$, XIA Shaoxia ${ }^{1}$, LI Ya $^{1,5}$, XU Ce $^{1,5}$ \& \\ YU Xiubo ${ }^{1,5 * *}$ \\ (1: Key Laboratory of Ecosystem Network Observation and Modeling, Institute of Geographic Sciences and Natural Resources \\ Research, Chinese Academy of Sciences, Beijing 100101, P.R.China) \\ (2: National Marine Environmental Monitoring Center, Dalian 116023, P.R.China) \\ (3: Key Laboratory of Sea Field Management Technology, State Oceanic Administration, Dalian 116023, P.R.China) \\ (4: Nanji Wetland National Nature Reserve Agency, Nanchang 330038, P.R. China) \\ (5: University of Chinese Academy of Sciences, Beijing 100049, P.R.China)
}

Abstract: This study aims to investigate the difference of releasing $\mathrm{C}, \mathrm{N}$ and $\mathrm{P}$ between wetland plant litter and overwintering migratory birds' droppings during their decomposition progresses and to investigate the influences of overwintering migratory birds' droppings on the decomposition of wetland plant litter. The decomposition bag technique and birds' droppings addition experiment were conducted from January to June, 2017 on the typical beaches of Lake Paying wetlands. Results showed significant differences

* 国家自然科学基金项目 (41471088,41701212)资助. 2018-10-09 收稿; 2018-11-19 收修改稿. 张全军( 1985 ), 男,博士研究生;E-mail: zhangqj.16b@igsnrr.ac.cn.

** 通信作者; E-mail: yuxb@igsnrr.ac.cn. 
of the dry matter loss rates, decomposition rates of three samples (plant litter mixture, wetland plant litter and birds' droppings) in different decomposition times. The mixture is highest, following by plant litter and birds' droppings. The addition of birds' droppings accelerated the decomposition process of plant litter and increased the average decomposition rate by $21.23 \%$. The simulation results of Olson negative exponential model estimated that it would cost 259, 314 and 355 days for decomposing 50\% of sample amount of plant litter mixture, wetland plant litter, droppings and about 3.1, 3.7 and 4.2 years for $95 \%$, respectively. The C, N and $\mathrm{P}$ of all the three samples showed net release pattern, with significant difference in relative return index. The carbon relative return index (CRRI) of plant litter mixture is highest, followed by the CRRI of wetland plant litter and birds' droppings. While the ranks of NRRI and PRRI (nitrogen and phosphorus) are both birds' droppings, plant litter mixture, and wetland plant litter. The differences of the stoichiometric ratio characteristics of the three samples were also significant. This study speculates that the addition of birds' droppings to the decomposition environment brings additional nutrients, which increases the number, type, activity of the decomposing microorganisms and the stimulation of birds' droppings to produce a series of degrading enzymes such as ligninase, cellulase, sucrase, etc., believed as the key factor for the acceleration of the decomposition process of plant litter. The initial nutrient contents and the changes of stoichiometric ratio of $\mathrm{C}, \mathrm{N}$ and $\mathrm{P}$ during the decomposition process are the dominant factors for the significant difference of decay rate and nutrient release.

Keywords: Lake Poyang; herbivorous overwintering migratory birds; wetland plants; Carex cinerascens Kükenth; decomposition carbon; nitrogen; phosphorus; biogeochemical cycle

湿地水鸟是指在生态上依赖于湿地栖息、取食、繁殖和越冬,即某一生活史阶段依赖于湿地,且在形态 和行为上对湿地形成适应特征的鸟类 ${ }^{[1]}$. 大多数水鸟是在全球范围内长距离迁徙的候鸟, 是湿地生态系统 的重要组分,对维护湿地生态系统平衡以及湿地物质和能量流动等方面起着非常关键的作用 ${ }^{[2]}$. 迁徙水鸟 往往栖息在湿地地区并形成大型水鸟集群,数量庞大水鸟群的取食和排泄活动对它们所栖息的湿地环境 ${ }^{[3]}$ 和湿地植物有着十分深远的影响 ${ }^{[4]}$, 特别是鸟类粪便为它们栖息的环境增加了大量外部营养来源 ${ }^{[3,5]}$, 它们 会加速湿地食物网流动进程从而加快湿地碳、氮、磷的循环速率 ${ }^{[6]}$. 然而, 关于湿地水鸟在这方面的定量研 究并不多见.

湿地鸟类粪便营养物的加人会导致湿地植物枯落物分解和养分释放过程的变化 ${ }^{[7]}$, 从而影响新生湿地 植物有效营养物的数量 ${ }^{[8]}$, 然后通过影响植物的萌发、生长、物种的丰度和地上生物量来影响植物群落的构 建和种群间对繁殖地的竞争 ${ }^{[9]}$. 而植物枯落物分解是调控湿地养分生物地球化学循环的关键环节, 也是维 持湿地生态系统能量流动、物质循环和养分平衡的重要生态过程 ${ }^{[10]}$. 但是目前关于湿地枯落物分解的研究 主要集中在分解过程中物质组分变化速率 ${ }^{[11-12]}$ 、元素释放动态 ${ }^{[12-14]}$ 以及对全球变化的响应 ${ }^{[15]}$ 等方面. 关于 湿地植物枯落物分解调控机制的研究大部分也只涉及到植物群落类型、枯落物本身的理化质量特征 ${ }^{[16]}$ 、分 解环境中的水文状况 ${ }^{[17-18]}$ 、温度状况、微生物状况 ${ }^{[19]}$ 等条件, 只有极少数的研究涉及到水鸟粪便对湿地植物 分解过程的影响 ${ }^{[7]}$, 关于水鸟粪便在湿地植物枯落物分解过程中的作用还知之甚少.

鄱阳湖湿地是东亚一澳大利亚西亚候鸟迁飞路线上最重要的越冬地, 是多种候鸟迁徙的必经之路与重 要栖息地 ${ }^{[20-21]}$. 水鸟调查数据表明,每年在鄱阳湖越冬的水鸟平均在 40 万只以上 ${ }^{[22]}$, 其中植食性雁鸭类的 种类多, 数量也是越冬水鸟中最大的,约占越冬候鸟总数的 $50 \%$ 以上 ${ }^{[23]}$. 数量庞大的水鸟在鄱阳湖湿地进 行取食、排泄以及栖息等越冬活动,每年冬天都会向湿地洲滩排泄大量的粪便. 这些粪便在鄱阳湖湿地洲滩 上分解过程有怎样的特征? 鸟粪的加人对湿地洲滩植物枯落物的分解过程又有什么样的影响? 这些十分 值得研究的问题在鄱阳湖湿地中的研究还比较罕见. 然而, 研究越冬的植食水鸟对湿地碳、氮、磷生物地球 化学循环过程的影响具有重要的科学理论意义, 对鄱阳湖湿地乃至长江中下游流域湖泊湿地保护与管理实 践具有重要的实际意义.

因此,本文的目的是: 1) 研究植食水鸟粪便分解过程和鄱阳湖湿地洲滩䔔草分解过程的差异及碳、氮、 磷释放动态特征;2) 研究植食水鸟粪便的加人对鄱阳湖湿地䔔草枯落物分解过程的影响. 


\section{1 材料和方法}

\section{1 研究区域概况}

本研究区域分布在鄱阳湖湿地的南矶山湿地国家级自然保护区典型的碟形湖泊白沙湖内 (图 1). 保护 区位于鄱阳湖主湖区南部,地处赣江北支、中支和南支汇人鄱阳湖开放水域冲积形成的三角洲前缘, 海拔在 $12 \sim 16 \mathrm{~m}$ ( 吴淞) 之间 ${ }^{[24]}$. 属亚热带暖湿型季风气候, 夏季炎热多雨, 冬季低温少雨. 受鄱阳湖季节性周期性 水文变化的影响,保护区内有明显的丰水期和枯水期. 丰水期内 (4-9 月), 保护区除总面积不足 $4 \mathrm{~km}^{2}$ 的南 山岛和矶山岛两个人居岛屿外其他草洲均被洪水淹没,处于典型的湖相水文状态. 枯水期内 (10 月至次年 3 月 ) 湖水消退归人河道和一些碟形洼地, 区内大小河流纵横, 碟形湖泊星罗棋布, 不同高程洲滩相继出露, 整 个三角洲地区呈现河、湖、洲交错的景观 ${ }^{[24]}$. 这种特殊的水文节律使得保护区内形成了大量土壤肥沃、水热 条件充裕的洲滩和浅滩, 发育了丰富的湿生植物和水生植物, 多样性极其丰富, 其中以荎草 (Carex cinerascens)、南荻 (Triarrhena lutarioriparia)、芦苇 (Phragmites australis) 等为优势种 ${ }^{[25]}$. 荎草是鄱阳湖湿地草洲分 布面积最广的湿生植物, 也是鄱阳湖湿地的优势植物, 分布遍及从湖岸到湖心水线之间全部洲滩 ${ }^{[25]}$, 因此本

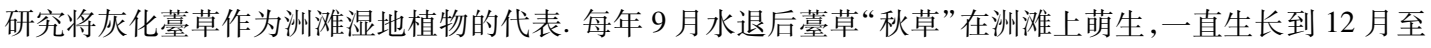
次年 3 月, 冬季地上部分逐渐开始枯萎分解; 1 月份过后在“秋草” 尚未全部死亡之前音草“春草”再次萌生, 一直生长到 4 月才逐渐被湖水淹没, 然后在水下休眠、死亡直至地上部分全部分解消失 ${ }^{[26]}$. 这种特殊的情况 为多种越冬珍禽和水鸟提供了良好的栖息地和食物来源, 使得鄱阳湖湿地成为亚洲最重要的珍禽越冬场 所,被誉为“白鹤王国”和“候鸟乐园”. 因此,鄱阳湖湿地是开展本研究的绝佳天然试验场.

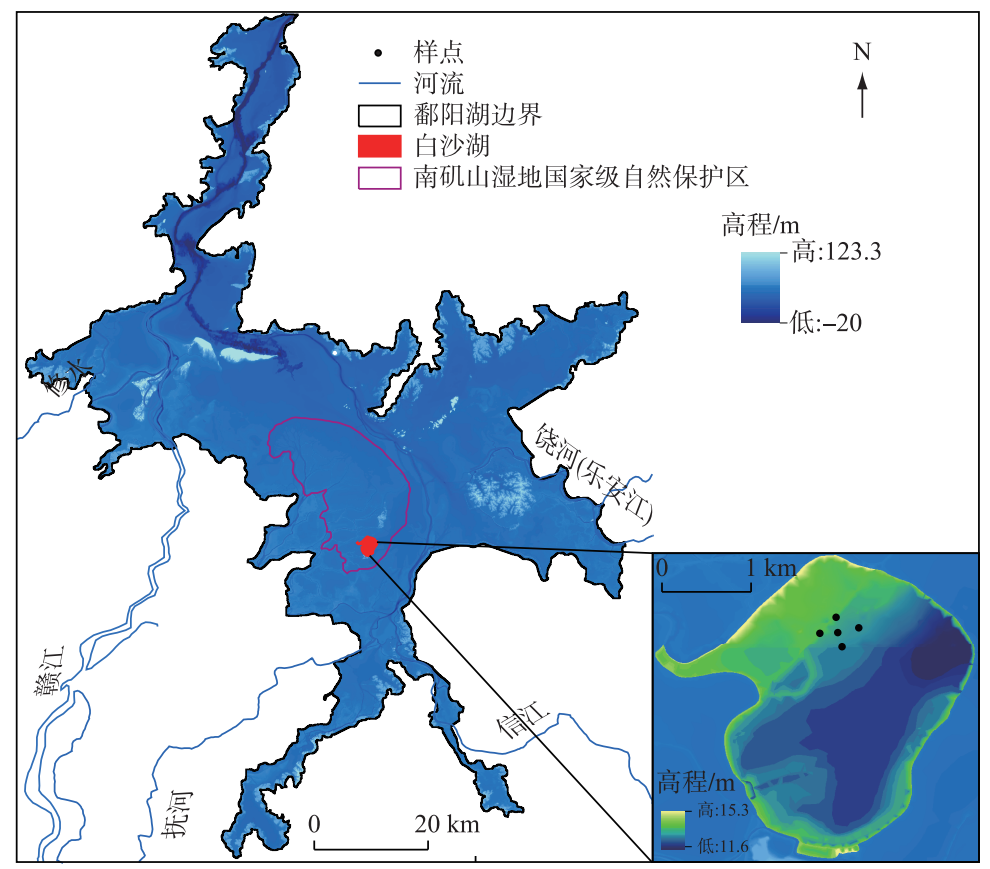

图 1 样点在鄱阳湖湿地中的位置

Fig.1 Position of sampling area in Lake Poyang Wetland

\section{2 野外试验设计与实验室分析}

本研究于 2017 年 1 月下旬在调查湿地植物和水鸟栖息地分布规律的基础上,在白沙湖内选择人为干扰 相对较少、植食性水鸟集聚频率较高的、鸟粪分布密集、植物群落发育良好的离湖心水线约 $200 \mathrm{~m}$ 的洲滩建 立 5 块固定试验样地, 样地中间约间隔 $50 \mathrm{~m}$, 样地内的优势植物为灰化薹草 (Carex cinerascens Kükenth), 盖 度在 $80 \% \sim 90 \%$ 之间, 高度为 $40 \sim 60 \mathrm{~cm}$, 薹草根部周围的土壤上或者荎草植株上分布有许多鸟粪. 在每个样 
地内选取一个点作为本次分解时间实验的 5 个重复样点(图 1).

分解模拟实验采用分解袋法. 选择 100 目 (孔径为 $0.15 \mathrm{~mm}$ )、规格为 $15 \mathrm{~cm} \times 20 \mathrm{~cm}$ 的白色网孔的尼龙网 袋作为分解袋. 这种网袋既能够避免分解袋中分解残体的非分解损失, 也能保证分解作用不限制. 在所设样 地附近小范围收割衰老荎草的叶片带回实验室用去离子水冲洗干净, 剪成 $10 \mathrm{~cm}$ 长小段后混合均匀 (消除 尺寸对分解的影响) 装人牛皮纸信封内, 置于烘箱 $120^{\circ} \mathrm{C}$ 杀青 $1 \mathrm{~h}$ 后再 $60^{\circ} \mathrm{C}$ 烘干至恒重. 同时在研究样地内 收集新鲜的越冬雁类粪便 (排泄在 $24 \mathrm{~h}$ 内, 以下简称鸟粪), 分批装人牛皮纸信封内, 置于 $60^{\circ} \mathrm{C}$ 烘箱内烘至 恒重. 分别取 $10.00 \mathrm{~g}$ 烘干的 6 个样品重复测量其初始养分含量(表 1). 再将剩下的样品称量 $10 \mathrm{~g}$ 分别装人 尼龙网袋中. 分解袋内的样品有 3 种形式:第 1 种形式是只装 $10 \mathrm{~g}$ 烘干的鸟粪, 第 2 种形式是只装 $10 \mathrm{~g}$ 烘干

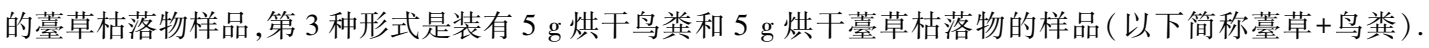
将制作好的 105 个分解袋带到样地用 PVC 管固定在预先设置的 5 个样点上,每个样点各放置 7 个 3 种不同 形式的样品袋 (不破坏地表原有枯落物结构), 将样品袋用短绳绑在插人泥土中的 PVC 管底部可以保证每 个样品袋都充分地接触地表且相互不挤压. 分别于实验开始后第 $5 、 15 、 30 、 60 、 90 、 120$ 和 150 天从各样点取 回分解袋. 试验终止于 6 月是因为洪水淹没了样地,改变了分解环境的水文条件.

将取回的分解袋带回实验室, 除去泥土、藻类以及吸附物等杂物, 再将菿草 + 鸟粪样品袋中的菫草残留 物单独挑出作为一类样品, 然后将所有样品装到有编号的牛皮信封中, 置于 $60^{\circ} \mathrm{C}$ 烘箱中烘干至恒重, 称量其 残余干物质质量后用球磨仪器将样品碾成粒径为 $0.06 \mu \mathrm{m}$ 的粉末, 然后分别密封于有编号的聚乙烯样品袋 中待测. 样品全碳 (TC) 和全氮 (TN) 含量使用元素分析仪 (Vario Max CN Analyzer, Elementar Analysensysteme GmbH, Germany) 测定; 全磷 (TP) 使用电感耦合等离子体发射光谱仪 (Inductively Coupled Plasma Optical Emission Spectrometer( ICP-OES), Optima 5300DV, Perkin-Elmer, America) 测定.

表 1 荎草枯落物和鸟粪样品初始养分含量

Tab.1 Abundances of C, N, P in initial Carex litter and birds' droppings

\begin{tabular}{ccccccc}
\hline 样品类型 & $\mathrm{C} / \%$ & $\mathrm{~N} / \%$ & $\mathrm{P} / \% 0$ & $\mathrm{C} / \mathrm{N}$ & $\mathrm{C} / \mathrm{P}$ & $\mathrm{N} / \mathrm{P}$ \\
\hline 荎草枯落物 & $43.08 \pm 0.277^{\mathrm{a}}$ & $1.15 \pm 0.060^{\mathrm{a}}$ & $0.97 \pm 0.019^{\mathrm{a}}$ & $37.46 \pm 0.001^{\mathrm{a}}$ & $4441.24 \pm 0.011^{\mathrm{a}}$ & $118.56 \pm 0.012^{\mathrm{a}}$ \\
鸟粪样品 & $36.82 \pm 1.308^{\mathrm{b}}$ & $1.33 \pm 0.072^{\mathrm{b}}$ & $2.44 \pm 0.093^{\mathrm{b}}$ & $27.68 \pm 0.002^{\mathrm{b}}$ & $1509.90 \pm 0.021^{\mathrm{b}}$ & $54.51 \pm 0.001^{\mathrm{b}}$ \\
\hline
\end{tabular}

* 不同小写字母表示在 $P<0.01$ 水平上差异显著,下同.

\section{3 数据处理与统计分析}

干物质损失率 ( the percentage of dry mass loss, $L_{t}$ ) 计算公式为 ${ }^{[13]}$ :

$$
L_{t}=\frac{M_{0}-M_{t}}{M_{0}} \times 100 \%
$$

式中, $L_{t}$ 表示时间 $t$ 时枯落物的损失率, $M_{t}$ 表示时间 $t$ 时枯落物干物质重量, $M_{0}$ 表示初始干物质重量, $t$ 为 枯落物分解进行的时间 (下文中含义相同).

干物质残留率 (the dry mass remaining, $r_{t}$ ) 计算公式为:

$$
r_{t}=100-L_{t}
$$

干物质瞬时分解速率 (the instantaneous loss rate of dry mass, $k$ ) 用 Olson 负指数衰减模型 ${ }^{[27]}$ 计算:

$$
M_{t}=M_{0} \mathrm{e}^{-k t}
$$

式中, $k$ 值越大表示枯落物的分解速度越快 (下文中含义相同).

干物质残余率 $(y)$ 用负指数衰减模型 ${ }^{[28]}$ Levenberg Marquardt 算法进行模拟:

$$
y=a \cdot \mathrm{e}^{-k t}
$$

式中, $y$ 为枯落物残留率, $a$ 为拟合参数, $\mathrm{e}$ 为自然底数.

相对养分归还指数 (relative return index, $R R I)$ 计算公式为 ${ }^{[13]}$ :

$$
R R I_{t}=\frac{M_{0} \cdot C_{0}-M_{t} \cdot C_{t}}{M_{0} \cdot C_{0}} \times 100 \%
$$


式中, $C_{t}$ 为 $t$ 时刻枯落物中某元素的浓度, $C_{0}$ 为枯落物中某元素的初始浓度. 用 $C R R I$ 表示碳的相对归还指 数 ( carbon relative return index), NRRI 表示氮的相对归还指数 (nitrogen relative return index),用 PRRI 表示磷 的相对归还指数 (phosphorus relative return index). 当 RRI 为正数时表示枯落物分解过程中元素发生了净释 放, 当 $R R I$ 为负数时则表示发生了净积累.

数据使用 Excel 2016 统计分析, 用 Origin9.0 制图, 在 SPSS 19.0 软件中进行单因素方差分析 (ANOVA) 和 LSD 多重比较的差异显著性检验.

\section{2 结果与分析}

\subsection{3 种样品干物质损失率、残留率和分解速率的分异特征}

分解时间对臺草枯落物干物质损失率的影响十分显著 $(F=102.931, P<0.0001$, 组间 $\mathrm{d} f=6$, 组内 $\mathrm{d} f=$ 98 ), 薹草枯落物、薹草+乌粪中薹草和鸟粪 3 种样品干物质损失率都随分解天数的增加而逐渐增大 (表 2).

分解 5 150 天薹草枯落物、薹草+乌粪中薹草和鸟粪的干物质损失率具有极显著的差异性 $(5$ 天: $F=$ $35.179, P<0.0001$; 15 天 : $F=21.930, P<0.0001$; 30 天 : $F=63.261, P<0.0001 ; 60$ 天: $F=7.869, P<0.005 ; 90$ 天 : $F=26.354, P<0.0001 ; 120$ 天: $F=34.880, P<0.0001 ; 15$ 天: $F=27.309, P<0.0001)$. 在分解的每个阶段总是薹 草 + 鸟粪中臺草损失率最高, 臺草样品次之, 鸟粪样品损失率最小. 分解 150 天后薹草 + 鸟粪中薹草、臺草和 鸟粪的干物质损失率依次为 $34.40 \% 、 29.64 \%$ 和 $26.20 \%$ (表 2).

表 2 薹草枯落物、臺草+乌粪中臺草和鸟粪样品分解过程中干物质损失率的动态变化

Tab.2 Dynamics of the percentage of dry mass loss during decomposition progress

\begin{tabular}{lcrrrrrc}
\hline \multirow{2}{*}{ 样品类型 } & \multicolumn{7}{c}{ 干物质损失率/\% } \\
\cline { 2 - 8 } & 5 天 & 15 天 & 30 天 & 60 天 & 90 天 & 120 天 & 150 天 \\
\hline 薹草样品 & $1.68 \pm 0.41^{\mathrm{a}}$ & $6.94 \pm 0.24^{\mathrm{a}}$ & $9.56 \pm 0.94^{\mathrm{a}}$ & $15.92 \pm 0.3^{\mathrm{ab}}$ & $18.76 \pm 0.33^{\mathrm{a}}$ & $23.62 \pm 2.61^{\mathrm{a}}$ & $29.64 \pm 0.24^{\mathrm{a}}$ \\
薹草+鸟粪中薹草 & $3.86 \pm 0.47^{\mathrm{b}}$ & $12.38 \pm 1.42^{\mathrm{b}}$ & $17.62 \pm 0.31^{\mathrm{b}}$ & $19.00 \pm 0.22^{\mathrm{bc}}$ & $24.78 \pm 0.62^{\mathrm{b}}$ & $30.40 \pm 0.92^{\mathrm{b}}$ & $34.40 \pm 0.75^{\mathrm{b}}$ \\
鸟粪样品 & $0.98 \pm 0.76^{\mathrm{a}}$ & $5.38 \pm 1.26^{\mathrm{a}}$ & $8.06 \pm 2.29^{\mathrm{a}}$ & $12.88 \pm 2.89^{\mathrm{a}}$ & $16.16 \pm 0.35^{\mathrm{a}}$ & $21.56 \pm 2.02^{\mathrm{a}}$ & $26.20 \pm 2.94^{\mathrm{c}}$ \\
\hline
\end{tabular}

通过 Olson 负指数衰减模型计算出每个分解时间点的瞬时衰减系数 $k$, 结果表明: 不同的分解时间对薹 草枯落物、臺草 + 鸟粪中臺草和鸟粪 3 种样品分解速率的影响极其显著 $(F=9.634, P<0.0001)$. 瞬时衰减系 数都表现出 $0 \sim 15$ 天内先快速增加至最大值, 其最大值分别为 $0.48 、 0.88$ 和 0.37 , 在 $30 \sim 60$ 天内瞬时衰减系 数都快速减小, $60 \sim 150$ 天内十分缓慢地减小直至稳定. 统计检验发现 3 种样品之间的瞬时衰减系数在 $0 \sim$ 150 天内的差异性极为显著 ( 5 天: $F=35.660, P<0.0001 ; 15$ 天: $F=22.271, P<0.0001 ; 30$ 天: $F=68.244, P<$ 0.0001 ; 60 天: $F=8.807, P=0.006$; 90 天: $F=27.215, P<0.0001 ; 120$ 天 : $F=37.234, P<0.0001 ; 150$ 天 : $F=$ $30.949, P<0.0001)$. 此外, 在每个分解时间点上瞬时衰减系数都是薹草 + 鸟粪中臺草最高, 薹草样品次之, 鸟 粪样品最小 (图 2A).

将实测数据计算出来的干物质残留率用 Olson 负指数衰减模型的 Levenberg Marquardt 算法进行拟合, 发现拟合效果非常好, 拟合系数 $R^{2}$ 均在 0.90 以上, 拟合方程的各参数如图 $2 \mathrm{~B} 、 \mathrm{C} 、 \mathrm{D}$ 与表 3 所示. 通过拟合方 程预测出薹草枯落物、薹草+乌粪中薹草和鸟粪样品分解完成 50\% 所需的时间大约依次为 314 、259 和 355 天，

表 3 薹草枯落物、臺草+乌粪中臺草和鸟粪样品分解过程 Olson 时间衰减模型拟合*

Tab.3 Fitting parameters of Olson decay model for samples during decomposition progress

\begin{tabular}{clcccc}
\hline 样品 & Olson 时间衰减模型 & $k$ & $R^{2}$ & $T_{0.5} / \mathrm{d}$ & $T_{0.95} / \mathrm{d}$ \\
\hline 薹草 & $R=98.09441 \mathrm{e}^{-0.001221 t}$ & 0.00221 & 0.94781 & 314 & 1356 \\
薹草+鸟粪中薹草 & $R=94.99457 \mathrm{e}^{-0.00268 t}$ & 0.00268 & 0.91101 & 259 & 1118 \\
鸟粪 & $R=98.83907 \mathrm{e}^{-0.00195 t}$ & 0.00195 & 0.95203 & 355 & 1536 \\
\hline
\end{tabular}

* 样品完成 $50 \%$ 分解所需时间 $T_{0.5}=-\ln (0.5) / k$, 完成 $95 \%$ 分解所需时间 $T_{0.95}=-\ln (0.05) / k$. 

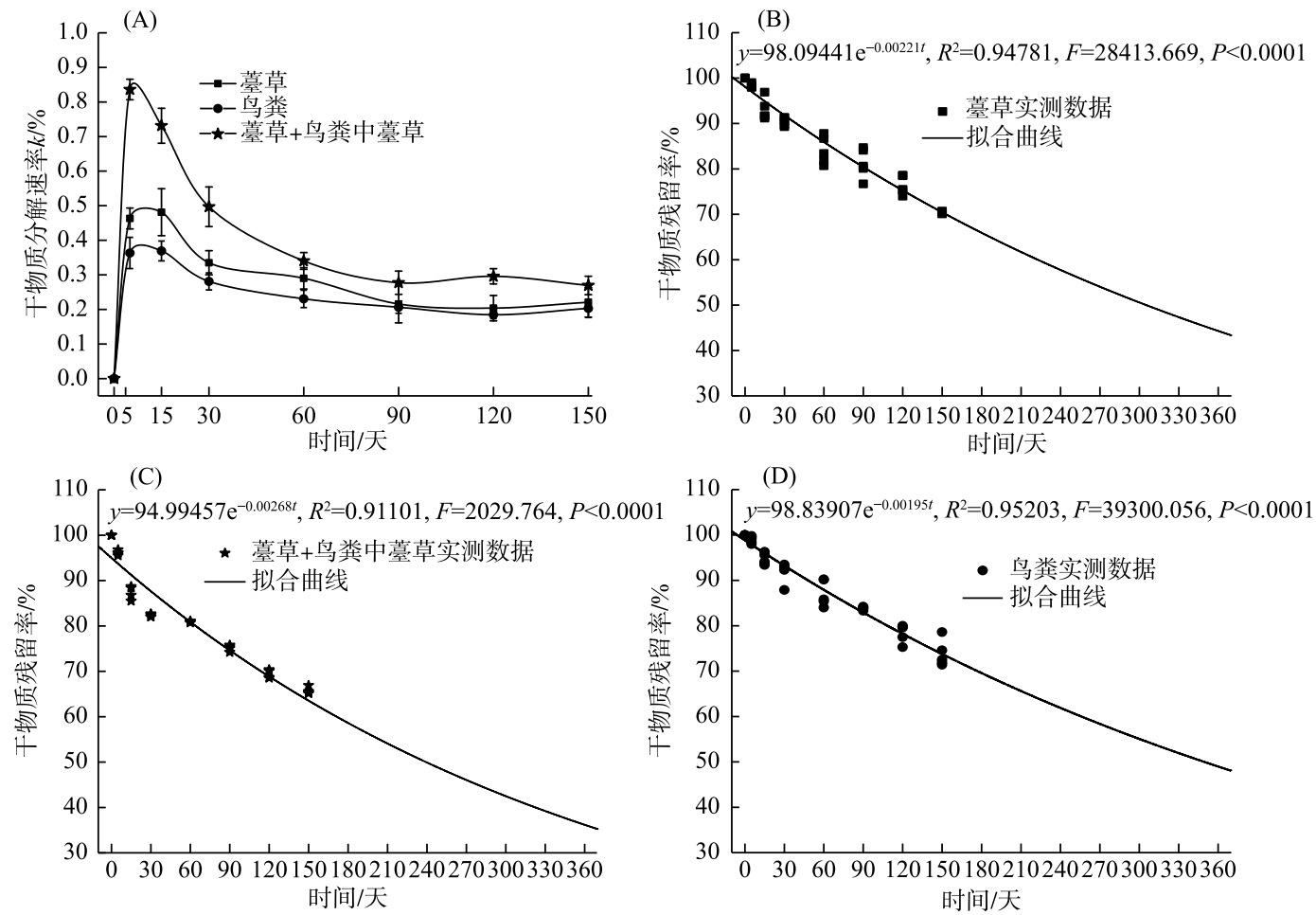

图 2 臺草枯落物、臺草+乌粪中臺草和鸟粪样品分解速率 $(\mathrm{A})$ 和干物质损失率 $(\mathrm{B} 、 \mathrm{C}$ 和 $\mathrm{D})$ 动态

Fig.2 Dynamics of decay rate (A) and the percentage of dry mass loss ( B, C and D) during decomposition progress

分解完成 $95 \%$ 所需的时间依次为 1356 天 (约 3.7 年)、1118 天 (约 3.1 年) 和 1536 天 (约 4.2 年), 并且 3 种样 品的平均分解速率差异极显著 $(P<0.0001)$, 分别为 $0.00221 、 0.00268$ 和 0.00195 .

\subsection{3 种样品碳、氮、磷相对归还指数分异特征}

在整个分解过程中萄草枯落物、菫草 + 鸟粪中荎草和鸟粪 3 种样品的碳、氮、磷元素都表现为净释放模 式 ( RRI>0; 图 3), 未出现积累的情况. 分解 $0 \sim 150$ 天 3 种样品的 $C R R I$ 值差异性并不显著 $(P>0.005)$, 但是 每个分解阶段菫草+鸟粪样品中荎草的 CRRI 值最高, 臺草样品次之, 鸟粪样品最小. 随着时间的推移 CRRI 持续增大. 到第 150 天时菫草+鸟粪中荎草、臺草和鸟粪 3 种样品的 CRRI 值次约为 $42.73 \% 、 34.91 \%$ 和 28.90\% (图 3A).

分解 $0 \sim 150$ 天 3 种样品的 $N R R I$ 值差异性极其显著 ( $F$ 为 $17.316 \sim 56.662, P<0.005$ ). 每个分解时间点 的 $N R R I$ 值均为鸟粪样品最高, 喜草+鸟粪中臺草次之, 荎草样品最小. 但是随着时间的推移 NRRI 值并不是 一直都升高, 而是波动起伏的. 菫草的样品的 $N R R I$ 值变化幅度很小, 最大值只有 $7.44 \%$, 并且一直处于小范 围波动状态; 薹草+鸟粪中蓋草和鸟粪样品的 $N R R I$ 值在 $0 \sim 30$ 天内快速升高至最大值, 而后再缓慢减小, 最 大值分别出现在菫草+鸟粪中薹草样品 (32.45\%) 和鸟粪样品 (61.84\%), 中间也偶尔有较小的起伏 (图 3B).

分解 $0 \sim 60$ 天 3 种样品的 $P R R I$ 值差异性并不显著 $(P>0.005)$, 但是 $90 \sim 150$ 天其差异性非常显著 $(F$ 为 $8.992 \sim 38.223, P<0.05)$. 每个分解阶段 $P R R I$ 值都是鸟粪样品最高, 荎草 + 鸟粪中荎草次之, 荎草样品最小. 薹草+鸟粪中薹草和鸟粪样品的 PRRI 值相差不大, 但是都比莒草的要高出很多. 随着时间的推移 3 种样品 的 PRRI 都持续升高, 直到第 150 天时鸟粪、荎草+鸟粪中薹草和薹草样品的 PRRI 值分别为 $61.63 \% 、 53.95 \%$ 和 $17.96 \%$ (图 3C).

\subsection{3 种样品碳、氮、磷化学计量学分异特征}

茥草枯落物、菫草+鸟粪中薹草和鸟粪 3 种样品的化学计量学性状动态变化十分明显 (图 4). 总的来 
看, 在分解的起始阶段, 不同样品的碳氮比值 $(\mathrm{C} / \mathrm{N})$ 大小为: 鸟粪样品 (约 27.68) <薹草样品 $($ 约 37.46) = 薹 草+鸟粪中薹草 (约 37.46). 随着分解的进行, 在 5 150 天内鸟粪的 $\mathrm{C} / \mathrm{N}$ 比一直比其他两种样品高很多, 而 分解 5 50 天左右薹草 + 鸟粪中荎草的 $\mathrm{C} / \mathrm{N}$ 一直高于薹草样品的, 但从 60 天以后, 薹草的 $\mathrm{C} / \mathrm{N}$ 就一直高于 薹草+乌粪中薹草的. 从样品种类来看, 臺草样品的 $\mathrm{C} / \mathrm{N}$ 在分解开始的前 5 天有小幅度的先上升后缓慢下降 趋势, 到第 150 天时下降至 24.71 , 比初始值低约 $34.03 \%$; 臺草+鸟粪中臺草的 $\mathrm{C} / \mathrm{N}$ 在 $0 \sim 5$ 天内迅速小幅度 下降后又迅速升高, 在第 15 天时达到最大值 (约 42.81), 而后逐渐缓慢下降, 到第 150 天时下降到 21.92 , 比 初始值低约 $36.94 \%$; 鸟粪样品的 $\mathrm{C} / \mathrm{N}$ 在 0 30 天持续升高到最大值(约 71.17), 然后持续下降, 到第 150 天 时变为 31.99 , 仍然比初始值高约 $15.57 \%$ (图 4A).
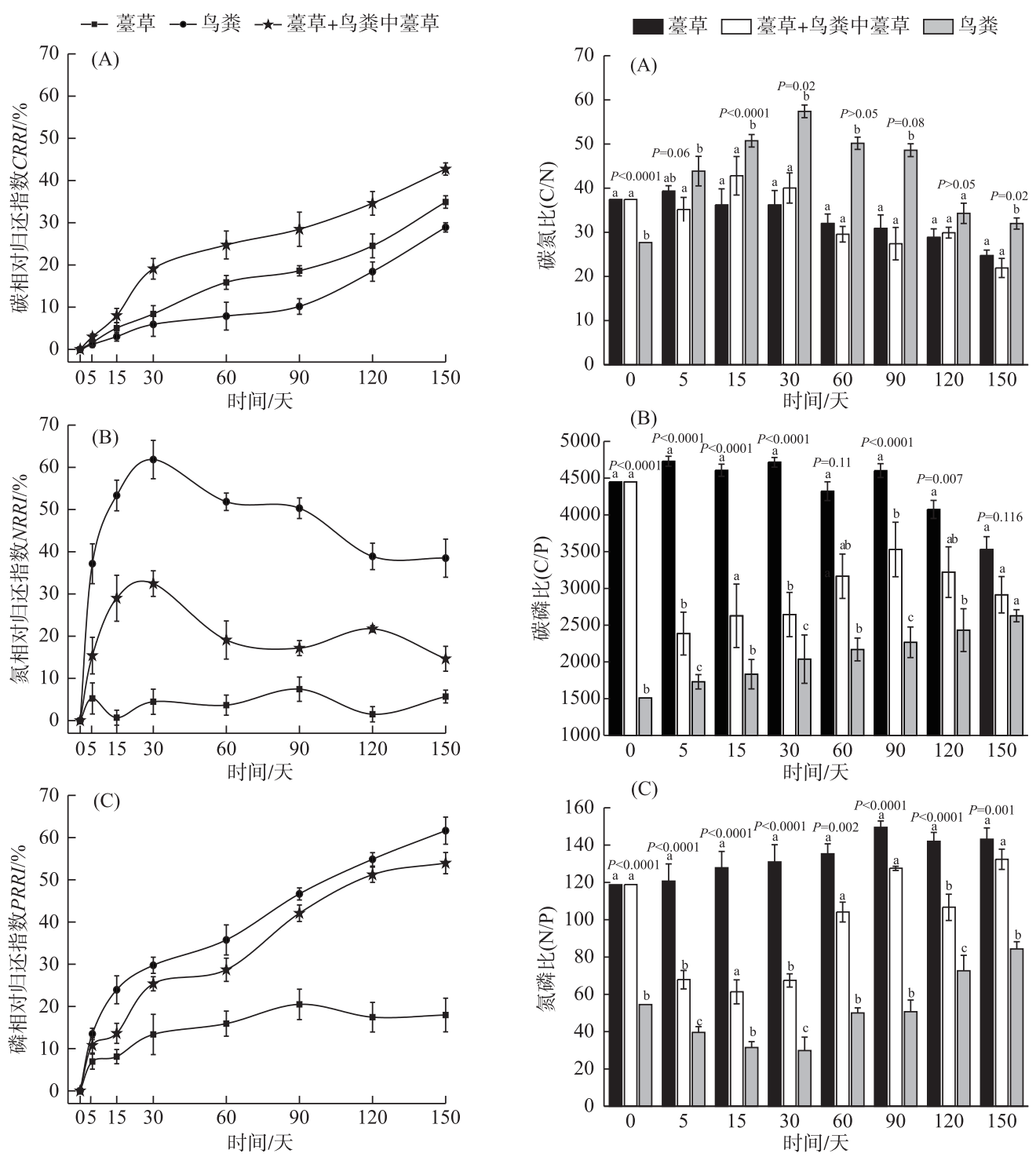

图 33 种样品分解过程中碳 (A)、 氮 $(N)$ 和磷 $(C)$ 相对归还指数动态

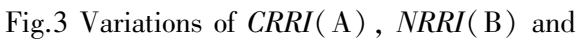
$\operatorname{PRRI}(\mathrm{C})$ during decomposition progress

图 4 样品分解过程中碳、氮、 磷的化学计量比动态

Fig.4 Variations of $\mathrm{C} / \mathrm{N}(\mathrm{A}), \mathrm{C} / \mathrm{P}(\mathrm{B})$ and $\mathrm{N} / \mathrm{P}(\mathrm{C})$ during decomposition progress 
3 种样品的碳磷比值 $(\mathrm{C} / \mathrm{P})$ 在 $0 \sim 90$ 天内存在十分显著的差异性 $(P<0.0001)$. 在分解的起始阶段, 不同 样品的 $\mathrm{C} / \mathrm{P}$ 为鸟粪样品 (约 1509) <薹草样品 (约 4441) $=$ 荎草 + 鸟粪中薹草样品 (约 4441). 在分解后的 5 150 天内三者的关系一直保持为鸟粪样品<薹草 + 鸟粪中臺草样品<蒙草样品. 臺草样品的 $\mathrm{C} / \mathrm{P}$ 虽在局部有 起伏,但总体呈缓慢下降趋势, 到第 150 天时比初始值低约 $20.56 \%$; 莒草+鸟粪中荎草的 $\mathrm{C} / \mathrm{P}$ 在 $0 \sim 5$ 天迅速 下降至最小值(约 2385), 之后在 5 90 天内缓慢上升至最大值(3529), 但仍未超过初始值, 之后又缓慢下降 至 2913 ; 鸟粪样品的 $\mathrm{C} / \mathrm{P}$ 呈逐渐缓慢升高的趋势,到第 150 天升高了约 $70.09 \%$ (图 4B).

3 种样品的氮磷比值 $(\mathrm{N} / \mathrm{P})$ 在 $0 \sim 150$ 天内都存在十分显著的差异性 $(P<0.0001)$. 在分解的起始阶段, 不 同样品的 $\mathrm{C} / \mathrm{P}$ 为鸟粪样品 (约 54.51) <薹草样品 (约 118.56) = 荎草 + 鸟粪中薹草 (约 118.56). 在分解后的 5

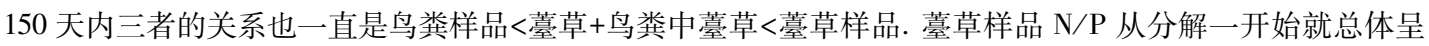
缓慢上升趋势, 到第 90 天时达到最大值(约 149.50 ), 增幅约为 $25.86 \%$, 之后逐渐缓慢下降, 到第 150 天时其值 约为 143.14 ; 荎草+鸟粪中薹草 $\mathrm{N} / \mathrm{P}$ 在前 15 天一直下降到最小值 (约 61.31), 之后快速上升, 在第 150 天时达 到最大值 (132.39), 比初始值增加了约 $11.46 \%$; 鸟粪样品的 N/P 一开始呈逐渐缓慢下降的趋势, 到第 30 天时 达到最小值 (29.85), 降幅约为 $45.24 \%$, 之后又快速地上升, 到第 150 天时已上升至 84.34 , 比初始值高 $54.72 \%$ (图 4C).

\section{3 讨论}

\section{1 乌粪的添加对湿地优势植物薹草枯落物分解过程的影响}

本研究结果表明不同的分解时间段内菫草枯落物、薹草 + 鸟粪中薹草和鸟粪 3 种样品的干物质损失率 和分解速率的差异性都十分显著 $(P<0.001)$. 干物质损失率都是随着分解时间的增加而不断升高, 而分解速 率在分解的前 $0 \sim 15$ 天内急速升高到最大值后开始缓慢降低至趋于稳定. 这与大多数湿地枯落物的分解过 程是一致的 ${ }^{[7,11,16]}$. 这可能是由于湿地环境中水分比较充足, 使得分解前期 3 种样品中的可溶解成分快速淋 溶, 水溶成分和非木质素碳水化合物优先分解, 且分解速率非常快 ${ }^{[12]}$, 而分解中后期已木质化的未分解碳水 化合物与原有木质素在微生物作用下同时降解, 这个过程就比较缓慢, 直到最后木质素含量趋于稳定, 残余 物质逐渐腐殖化,分解速率较小且几乎不变 ${ }^{[29]}$.

本研究结果还表明, 鸟粪添加对菿草枯落物分解过程有很大的影响, 无论是干物质损失率还是分解速 率在 3 种样品之间都有非常显著的差异性. 在整个分解过程中二者数值都表现为薹草+乌粪中薹草样品 $>$ 菫 草样品>鸟粪样品, 用模型模拟也得到了相同的结果 (表 3). 这说明鸟粪添加改变了枯落物的成分, 能够加 速菫草枯落物的分解进程. 这与 Lee 等 ${ }^{[7]}$ 在水稻田开展的控制试验研究结果类似,他们发现添加 $80 \mathrm{~g}$ 鸟粪 处理水稻秸秆的分解率显著高于添加 $40 \mathrm{~g}$ 鸟粪处理, 而添加 $40 \mathrm{~g}$ 鸟粪处理的分解速率又高于对照组 (未添 加鸟粪) 的. 这可能是由于水鸟粪便的加人增加了额外的营养物质, 对荎草枯落物的分解产生了积极的刺激 作用 ${ }^{[8]}$, 这种刺激作用又进一步增加了分解环境中有效养分的供应. 分解环境中养分供应会影响异养分解 者对营养物质的可获得性,通常被认为是控制分解速率的一个重要因素 ${ }^{[30]}$. 一般情况下, 枯落物分解过程 中, 枯落物营养含量不足以满足异养生物分解者活动对营养物质的需求量 ${ }^{[31]}$. 所以本研究推测鸟粪添加一 方面给分解环境中带来额外的营养物质可能成为了枯落物中微生物分解者的重要营养补给, 使薹草 + 鸟粪 样品中分解者微生物的数量、种类以及活性增强. 另一方面, 可能是鸟粪的增加刺激产生了一系列降解酶, 如木质素酶、纤维素酶、蔗糖酶等, 胞外酶数量和活性增加, 从而导致出苔草+乌粪样品中的苔草分解速率 更高.

荎草枯落物的分解速率高于鸟粪可能与分解过程 $\mathrm{C} / \mathrm{N}$ 变化有关. 在湿地环境中, $\mathrm{C} / \mathrm{N}$ 一般被认为是表征 分解速率的最重要指标 ${ }^{[12]}$, 大量研究表明 $\mathrm{C} / \mathrm{N}$ 较高的枯落物分解速率较低 ${ }^{[13,16-17]}$. 本研究中, 在分解的起始 阶段, 鸟粪样品的 $\mathrm{C} / \mathrm{N}$ 比菫草样品的低, 但是随着分解的进行, 在 5 150 天内鸟粪样品的 $\mathrm{C} / \mathrm{N}$ 一直比莒草样 品的要高出很多 (图 4A), 直接减缓了乌粪的分解速率. 在 $\mathrm{C} / \mathrm{N}>30$ 的时候, $\mathrm{N}$ 将成为分解过程中的限制性因 素, 微生物分解活动也会因 $\mathrm{C} / \mathrm{N}$ 的增高而变慢 ${ }^{[32]}$. 这个现象的另外一个原因可能是水鸟在取食蕚草后将一些 易分解的成分消化利用,将难分解的成分留在鸟粪之中,不过这个原因有待进一步研究验证.

\section{2 乌粪添加对湿地优势植物薹草枯落物分解过程中碳、氮、磷释放的影响}

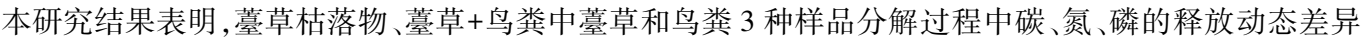


非常明显, 并且在整个分解阶段都表现为净释放模式. 在整个分解过程中, 3 种样品的 CRRI 值都持续升高, 并且表现为薹草 + 鸟粪中臺草样品 > 臺草样品 >鸟粪样品 (图 3A); NRRI 值是高低波动起伏的, 并且整体表现

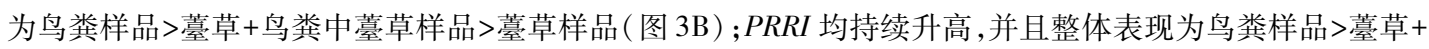
鸟粪中荎草样品>薹草样品 (图 3C). 这与 Zhao 等 ${ }^{[13]}$ 在滨海湿地、Sun 等 ${ }^{[17]}$ 在三江平原沼泽湿地、Lan 等 ${ }^{[16]}$ 在白洋淀湿地以及张广帅等 ${ }^{[14]}$ 在鄱阳湖湿地枯落物分解中养分释放动态模式相似.

一般情况下, 碳归还指数变化整体包含快速增加和缓慢增加两个过程 ${ }^{[13]}$. 在淋溶过程中溶解性碳水化 合物快速发生淋溶, 碳归还指数会快速升高 ${ }^{[16]}$, 后面受外部环境因子的激发作用或者外源营养元素输人的 影响, 分解速率加快, 土壤微生物数量变多、活力增强, 分解残留物中剩余木质素、纤维素和单宁等难分解物 质开始逐渐被微生物所利用, 此时碳归还指数的增加速度开始减缓甚至开始降低 ${ }^{[12]}$. 前文已经叙及鸟粪的 加人促进了荎草枯落物的分解, 这可能是造成荎草 + 鸟粪中荎草 $\mathrm{C}$ 释放速度比菫草枯落物高的关键原因.

然而, 在本研究中对于氮归还指数来说, 在整个分解过程中并不是一直呈升高的趋势, 而是有升有降, 但 整体表现为净释放模式. 尽管在淋溶过程中氮会由于含氮盐的流失而快速减少,但是氮素多以复杂且不易被 淋溶的大分子蛋白质存在, 并且其分解主要受微生物的影响, 微生物在分解这些大分子蛋白质时对氮需求的 增加会导致氮固定, 氮的归还指数升高会减缓甚至会大幅下降 ${ }^{[33]}$, 微生物固氮作用是分解过程中氮元素积累 的主要原因 ${ }^{[34]}$. 可能由于鸟粪结构比较松散, 可溶性氮含量较高, 容易受湿地水分淋溶的影响, 所以鸟粪样品 氮的释放速度一直比其他两种样品要快, 并且这种 $\mathrm{N}$ 淋溶的速度远远抵消了荎草分解速率较快所增加的氮释 放量. 但是臺草+鸟粪中荎草氮的释放速度比臺草枯落物高很多可能主要还是分解速率较高造成的.

磷的归还变化受物理和生物双重因素的影响, 分解初期磷的淋溶作用比较强, 分解残留物中磷归还速 度非常高, 当释放到一定程度时, 归还速度就趋于稳定 ${ }^{[35]}$, 这可能是因为在植物组织中的磷主要以磷酸根或 化合物的形式存在而容易发生淋溶损失. 而分解后期微生物降解过程中, 当微生物的营养需求不受磷限制 时, 过量的磷会限制微生物的活性 ${ }^{[8,36]}$. 鸟粪中磷的含量远远高于荎草枯落物中磷的含量, 但是鸟粪结构十 分松散, 磷的淋溶流失速率远远要高于洜草枯落物的, 所以分解速率所带来的磷释放的增加量已经被快速 的淋溶量所抵消, 因此造成了乌粪的 PRRI 值比荎草枯落物的要高很多的现象. 但是荎草+乌粪中薹草磷的 释放速度比瞢草枯落物高还可能仍然是由分解速率较高引起的.

\section{4 结论}

在鄱阳湖湿地枯水期植食越冬候鸟粪便和典型湿地植物莒草枯落物模拟分解试验得到以下结论：

1) 鄱阳湖湿地枯水期植食越冬候鸟粪便的加人对典型湿地植物枯落物的分解有显著促进作用, 分解 150 天后䔔草+鸟粪中荎草、菫草和鸟粪样品干物质损失率依次为 $34.40 \% 、 29.64 \%$ 和 $26.20 \%$. Olson 负指数 衰减模型得出鸟粪的加人使荎草的平均分解速率提升了 $21.23 \%$ 左右. 本研究推测鸟粪添加给分解环境带 来额外的营养物质, 使分解者微生物数量、种类、活性增加以及鸟粪刺激产生了一系列降解酶, 如木质素酶、 纤维素酶、蔗糖酶等, 胞外酶数量和活性增加, 是鸟粪促进莒草分解的关键因素, 而样品中初始养分含量以 及分解过程碳、氮、磷化学计量比的变化是造成其分解速率和养分释放显著性差异的主要原因.

2) Olson 负指数衰减模型的 Levenberg Marquardt 算法能很好地拟合 3 种样品的分解过程, 拟合系数 $R^{2}$

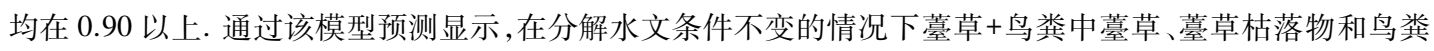
样品分解完成 50\%所需的时间分别为 259 、314 和 355 天左右, 分解完成 $95 \%$ 所需的时间依次约为 3.1、3.7 和 4.2 年.

3) 荎草+乌粪中荎草、薹草枯落物和鸟粪样品分解过程中的碳、氮、磷都表现为净释放模式, 随着分解过 程的进行 $R R I$ 整体在持续升高, 并且其养分的 $R R I \mathrm{~s}$ 之间差异也十分显著. CRRI 值表现为薹草 + 鸟粪中薹

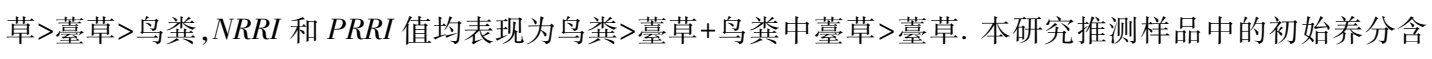
量以及分解过程碳、氮、磷化学计量比的变化是造成其分解速率和养分释放差异显著的重要原因.

致谢: 感谢江西鄱阳湖南矶湿地国家级自然保护区管理局及相关工作人员对本研究野外工作的配合与支 持; 感谢南昌大学生命科学研究院流域生态学研究所的金松斌老师、兰志春博士、沈瑞昌博士在室内试验中 提供的便利与帮助; 感谢南昌大学张欢老师、孟竹剑同学、吴申浩同学和王拿同学、江西师范大学研究生饶 
滴滴同学在野外样地布设、取样以及作图过程中的帮助; 感谢荷兰瓦格宁根大学( Wageningen University and Research) 窦月含博士对英文摘要的润色修改.

\section{5 参考文献}

[ 1 ] Nisbet ICT. Waterbird population estimates: fourth edition. Waterbirds, 2013, 31(1) : 152-153.

[ 2 ] Sekercioglu CH. Increasing awareness of avian ecological function. Trends in Ecology \& Evolution, 2006, 21 ( 8): 464-471.

[ 3 ] Manny BA, Johnson WC, Wetzel RG. Nutrient additions by waterfowl to lakes and reservoirs: Predicting their effects on productivity and water quality. Hydrobiologia, 1994, 279/280(1) : 121-132.

[ 4 ] Tomassen HBM, Smolders AJP, Lamers LPM et al. How bird droppings can affect the vegetation composition of ombrotrophic bogs. Canadian Journal of Botany, 2005, 83(8) : 1046-1056.

[ 5 ] Chaichana RRL, Moss B. Birds as eutrophicating agents: a nutrient budget for a small lake in a protected area. Hydrobiologia, 2010, 646(1): 111-121.

[ 6 ] Bauer HSS, Klaassen M. Quantification of allochthonous nutrient input into freshwater bodies by herbivorous waterbirds. Freshwater Biology, 2010, 53(1) : 181-193.

[ 7 ] Lee GM, Kim HT, Kim JG. Effects of wintering waterfowl's feces on nutrient dynamics of paddy fields and rice growth. Journal of Ecology \& Environment, 2012, 35(4) : 291-299.

[ 8 ] Elwood J, Newbold J, Trimble A et al. Limiting role of phosphorus in a woodland stream ecosystem: Effects of P enrichment on leaf decomposition and primary producers. Ecology, 1981, 62(1) : 146-158.

[ 9 ] Xiong SJ, Nilsson C. The effects of plant litter on vegetation: a meta-analysis. Journal of Ecology, 1999, 87 (6) : 984-994.

[10] Mitsch WJ, Gosselink JG eds. Wetlands: 5th edition. United States: John Wiley \& Sons, Inc, 2015.

[11] Zhang GW, Yu XB, Gao Y et al. Effects of water table on cellulose and lignin degradation of Carex cinerascens in a large seasonal floodplain. Journal of Freshwater Ecology, 2018, 33(1) : 311-325.

[12] Iii SED, Corronado-Molina C, Childers DL et al. Temporally dependent C, N, and P dynamics associated with the decay of Rhizophora mangle L. leaf litter in oligotrophic mangrove wetlands of the southern everglades. Aquatic Botany, 2003, 75 (3) : 199-215.

[13] Zhao QQ, Bai JH, Liu PP et al. Decomposition and carbon and nitrogen dynamics of Phragmites australis litter as affected by flooding periods in coastal wetlands. Clean-Soil, Air, Water, 2015, 43(3): 441-445.

[14] Zhang GS, Yu XB, Liu Y et al. Accumulation effect of litter decomposition and water level on carbon and nitrogen in shallow lake water of Lake Poyang. J Lake Sci, 2018, 30(3) : 668-679. DOI: 10.18307/2018.0309. [张广帅, 于秀波, 刘 宇等. 鄱阳湖碟形湖泊植物分解和水位变化对水体碳、氮浓度的叠加效应. 湖泊科学, 2018, 30(3): 668-679.]

[15] Peter M, Schile-Beers LM, Mozdzer TJ et al. Global-change effects on early-stage decomposition processes in Tidal Wetlands-Implications from a global survey using standardized litter. Biogeosciences, 2018, 15(10) : 3189-3202.

[16] Lan Y, Cui BS, You ZY et al. Litter decomposition of six macrophytes in a eutrophic shallow lake (Baiyangdian Lake, China). Clean-Soil, Air, Water, 2012, 40(10) : 1159-1166.

[17] Sun ZG, Mou XJ, Liu JS. Effects of flooding regimes on the decomposition and nutrient dynamics of Calamagrostis angustifolia litter in the Sanjiang Plain of China. Environmental Earth Sciences, 2012, 66( 8) : 2235-2246.

[18] Langhans SD, Tockner K. The role of timing, duration, and frequency of inundation in controlling leaf litter decomposition in a river-floodplain ecosystem (Tagliamento, Northeastern Italy). Oecologia, 2006, 147(3) : 501-509.

[19] Verónica F, Chauvet E. Synergistic effects of water temperature and dissolved nutrients on litter decomposition and associated fungi. Global Change Biology, 2011, 17(1) : 551-564.

[20] Ding XS. Protection and use of ecotourism resource of Poyang Lake Nature Reserve. Jiangxi Forestry Science and Technolo$g y, 2003,30(6)$ : 30-34. [丁贤生. 鄱阳湖自然保护区湿地生态旅游资源的保护与利用. 江西林业科技, 2003,30 (6) : 30-34.]

[21] Xia SX, Liu GH, Yu XB et al. Importance assessment of wintering habitats for migratory waterfowl in Lake Poyang. J Lake Sci, 2015, 27(4) : 719-726. DOI: 10.18307/2015.0421. [夏少霞, 刘观华, 于秀波等. 鄱阳湖越冬水鸟栖息地评 
价. 湖泊科学, 2015, 27(4): 719-726.]

[22] Li FS ed. Ecological study on wetlands and waterbirds in Poyang Lake. Beijing: Popular Science Press, 2011. [ 李凤山. 鄱阳湖湿地和水鸟的生态研究. 北京: 科学普及出版社, 2011.]

[23] Tu YG, Yu CH, Huang XF et al. Distribution and population of the overwintering anatidae waterfowl in the Poyang Lake. Acta Agriculturae Universitatis Jiangxiensis, 2009, 31(4): 760-764. [涂业苟, 俞长好, 黄晓凤等. 鄱阳湖区域越冬雁 鸭类分布与数量. 江西农业大学学报, 2009, 31(4) : 760-764.]

[24] Liu XZ, Hu BH eds. Comprehensive and scientific survey of Jiangxi Nanjishan Wetland Nature Reserve. Beijing: China Forestry Publishing House, 2005. [刘信中, 胡斌华. 江西南矶山湿地自然保护区综合科学考察. 北京: 中国林业出 版社, 2005.]

[25] Zhang QJ, Yu XB, Qian JX et al. Distribution characteristics of plant communities and soil organic matter and main nutrients in the Poyang Lake Nanji Wetland. Acta Ecologica Sinica, 2012, 32(12): 3656-3669. [张全军, 于秀波, 钱建金金 等. 鄱阳湖南矶湿地优势植物群落及土壤有机质和营养元素分布特征. 生态学报, 2012, 32(12): 3656-3669.]

[26] Zhang QJ, Yu XB, Hu BH. Research on the characteristics of plant communities in the Poyang Nanji Wetlands. Resources Science, 2013, 35(1) : 42-49. [张全军, 于秀波, 胡斌华. 鄱阳湖南矶湿地植物群落分布特征研究. 资源科学, $2013, \mathbf{3 5}(1): 42-49$. ]

[27] Olson JS. Energy storage and the balance of producers and decomposers in ecological systems. Ecology, 1963, 44(2): 322-331.

[28] Lang WGE. A critique of the analytical methods used in examining decomposition data obtained from litter bags. Ecology, $1982,63(6): 1636$.

[29] Kalbitz K, Kaiser K, Bargholz J et al. Lignin degradation controls the production of dissolved organic matter in decomposing foliar litter. European Journal of Soil Science, 2006, 57(4) : 504-516.

[30] Debusk WF, Reddy KR. Litter decomposition and nutrient dynamics in a phosphorus enriched everglades marsh. Biogeochemistry, 2005, 75(2): 217-240.

[31] Enríquez S, Duarte CM, Sand-jensen K. Patterns in decomposition rates among photosynthetic organisms: the importance of detritus C:N:P content. Oecologia, 1993, 94(4): 457-471.

[32] Atkinson RB, Cairns J. Plant decomposition and litter accumulation in depressional wetlands: functional performance of two wetland age classes that were created via excavation. Wetlands, 2001, 21(3) : 354-362.

[33] Wu HT, Lv XG, Yang Q et al. Early-stage litter decomposition and its influencing factors in the wetland of the Sanjiang Plain, China. Acta Ecologica Sinica, 2007, 27(10): 4027-4035. [武海涛, 吕宪国, 杨青等. 三江平原典型湿地枯落 物早期分解过程及影响因素. 生态学报, 2007, 27(10): 4027-4035.]

[34] Aber JD, Melillo JM. Litter decomposition: measuring relative contributions of organic matter and nitrogen to forest soils. Can J Bot, 1980, 58(4) : 416-421.

[35] Wrubleski DA, Murkin HR, Valk AGVD et al. Decomposition of emergent macrophyte roots and rhizomes in a Northern Prairie Marsh. Aquatic Botany, 1997, 58(2) : 121-134.

[36] Xie YH, Yu D, Ren B. Effects of nitrogen and phosphorus availability on the decomposition of aquatic plants. Aquatic Botany, 2004, 80 (1) : 29-37. 